DOI: $10.35381 /$ r.k.v4i1.374

\title{
El Balanced Scorecard como herramienta de control interno en el sector Manufacturero
}

\section{The Balanced Scorecard as an internal control tool in the sector manufacturer}

\author{
Narcisa Senaida Lazo Torres \\ nlazot@psg.ucacue.edu.ec \\ Universidad Católica de Cuenca \\ Ecuador \\ https://orcid.org/0000-0002-6021-4014 \\ Juan Carlos Erazo Álvarez \\ jcerazo@ucacue.edu.ec \\ Universidad Católica de Cuenca, Posgrado \\ Ecuador \\ https://orcid.org/0000-0001-6480-2270 \\ Cecilia Ivonne Narváez Zurita \\ inarvaez@ucacue.edu.ec \\ Universidad Católica de Cuenca \\ Ecuador \\ https://orcid.org/0000-0002-7437-9880
}

Recibido: 10 de agosto de 2019

Aprobado: 30 de agosto de 2019

\section{RESUMEN}

En el mundo empresarial existen herramientas gerenciales que le permiten controlar y evaluar con rigor sus procesos administrativos, por tal razón la presente investigación se desarrolló con el objetivo de implementar una herramienta eficaz para mejorar el control interno de la empresa Indalum, llegando a determinar que el modelo Balanced scorecard es adecuado para este fin, ya que en la actualidad se ha convertido en el sistema de gestión estratégica que más abarca al mundo organizacional, siendo transcendental desarrollar a través de sus cuatro perspectivas: financiera, clientes, procesos y aprendizaje. Además, se estableció indicadores de seguimiento y control para los diferentes au- 
tores que intervienen en todos los procesos, especialmente en las áreas críticas, inventarios, cuentas por cobrar, proceso de fabricación, identificándose como el mayor problema la falta de conocimiento de los manuales y procedimientos y la deficiente comunicación del personal.

Descriptores: Balanced scorecard; Control interno; Eficaz; Indicadores; Procesos.

\section{ABSTRACT}

In the business world there are management tools that allow you to control and rigorously evaluate your administrative processes, for this reason this research was developed with the objective of implementing an effective tool to improve the internal control of the Indalum Company, reaching to determine that the Ba-lanced scorecard model is suitable for this purpose, since it has now become the strategic management system that most encompasses the organizational world, being transcendental to develop through its four perspectives: financial, clients, processes and learning. In addition, monitoring and control indicators were established for the different authors involved in all processes, especially in the critical areas, inventories, accounts receivable, manufacturing process, identifying the lack of knowledge of manuals and procedures as the biggest problem and poor staff communication

Descriptors: Balanced scorecard; Internal control; Effective; Indicators; Processes.

\section{INTRODUCCIÓN}

La manufactura es uno de los sectores más importantes para un país, ya que mediante la elaboración de bienes que generan altos índices de beneficios económicos, aportados con valor de diferenciación a costos bajos y abasteciendo a mercados populares en donde mayor esta la población consumidora. El desarrollo de este sector ha logrado reforzar la economía del Ecuador, como indica las estadísticas registradas en el INEC a septiembre de 2018 dicha actividad a generado el 11\% del empleo total del Ecuador.

La participación de utilidades del sector Manufacturero en la categoría productos metálicos a nivel ciudad, indica para el año 2015 Quito obtuvo $\$ 20,25$ millones de USD con $64.61 \%$ de participación, Guayaquil $\$ 7,35$ millones de USD y $23.45 \%$ de participación, Cuenca $\$ 1,20$ millones de USD y 3.84\% de participación seguida de Puerto Francisco de 
Orellana (Coca) con $\$ 0,62$ millones de USD y $1.99 \%$ y por último a Eloy Alfaro con $\$ 0,59$ millones de USD y $1.87 \%$ de participación.

Sin embargo, cabe recalcar que esta categoría de fabricación de productos de aluminio aptos para el uso doméstico aporta con $\$ 3,35$ millones de USD resultado ser $10.99 \%$ de participación en la economía del país.

La Unidad de análisis de la presente investigación es la empresa Indalum S. A. que fue creada en 1978 con el objetivo principal de fabricar artículos de aluminio para el hogar, está ubicada en la Av. Gil Ramírez Davalos 4-18 y Francisco Pizarro de la ciudad de Cuenca. Actualmente existen dos empresas UMCO e INDALUM que ocupan el primer y segundo lugar respectivamente en el mercado ecuatoriano, dándose este posicionamiento de mercado por aproximadamente 40 años de trayectoria.

El segmento de mercado al cual se dirige es clase media baja, ya que los productos que fabrica, es de muy bajo costo, la distribución es a nivel Nacional con mayor aceptación en la Costa y Sierra. Logrando convertir en principales clientes de INDALUM a los almacenes de cadena, quienes requieren productos como: ollas, sartenes, pailas, lecheros, bandejas, etc., buscando bajos precios, tiempo de respuesta oportuna y buena calidad; otro grupo de cliente son los distribuidores quienes ofrecen nuestros productos a subdistribuidores, mayoristas y recorredores para la venta al consumidor final.

En los últimos 5 años la empresa ha reflejado un crecimiento del 12\% promedio anual en los ingresos; sin embargo, la administración no ha demostrado buen desempeño, reflejando deficiencias en el control interno de los procesos entre las áreas: Administración, Ventas y Producción; generando pérdidas económicas a la empresa.

Se puede mencionar algunos de las causas:

- Creación de clientes sin aplicar las políticas de procedimientos de la empresa, generando cartera vencida y cuentas incobrables, dando como resultado pérdidas económicas.

- Omisión de auditorías a los saldos de las cuentas por cobrar generando alto riesgo de no recuperar las cuentas por cobrar. 
- Falta de control de los inventarios, reflejando diferencias entre los saldos físicos vs montos contables.

- Inadecuado manejo de los recursos: suministros, Personal y demás materiales de la empresa, generando mayor costo y gastos.

- Falta de responsabilidad del personal que desempeña las tareas.

- El personal no tiene la capacitación adecuada sobre sus funciones.

Por tal motivo se ve la necesidad de desarrollar esta investigación para proponer un control interno óptimo y lograr los objetivos estratégicos a través del Balanced Scorecard en la empresa Indalum, y así evitar pérdidas económicas.

\section{Herramientas de control interno de la organización empresarial.}

Es fundamental mencionar la importancia de los procesos de la gestión administrativa de las empresas logrando entender la necesidad de desarrollar herramientas para mejorar partiendo desde los altos mandos hasta la aplicación en cada uno de los empleados y lograr concientizar las responsabilidades que ejercen en cada actividad y las consecuencias ya sean positivas o negativas siempre terminaran reflejando en los resultados de los logros o desaciertos de los objetivos y metas de la empresa.

Según las teorías de varios autores con el afán de mejorar el proceso de gestión administrativa se han creado herramientas que ayudan a llegar a los objetivos de la empresa, siendo uno de ellos el Cuadro de Mando Integral conocido también como Balanced scorecard y su implementación se considera en cualquier proyecto como una oportunidad de desarrollo, dentro del sistema económico global para las empresas Pymes, esto permite de acuerdo a Martínez \& El Kadi (2019), plantean que esto permite promover la calidad total de la empresa por cuanto se miden los procesos para trabajar en la mejora continua. "La existencia de Pymes eficientes es vital para un programa de industrialización de un país, pues desempeñan, entre otros, el papel de proveedoras de las piezas y componentes necesarios para las grandes industrias y contribuyen al empleo y a la capacitación de 
trabajadores y empresarios"; según afirma el ex director de la Organización de las Naciones Unidas para el Desarrollo Industrial (ONUDI), Mauricio de María y Campos. En (Manzo, 2013, p. 11)

\section{Cuadro de Mando Integral o Balanced Scorecard}

En esta trayectoria de evolución del Balanced scorecard como modelo de dirección y gestión empresarial se considera cuatro etapas: la primera etapa es la que identifica el desempeño de la empresa, mediante los distintos indicadores que nos permitan medir su alcance de la gestión administrativa. Siguiente etapa el Balanced scorecard incluye la visión estratégica. Pasando a la fase donde se unifican los objetivos con los indicadores, a partir de un análisis causa-efecto y se establecen los mapas estratégicos. Por último, la cuarta etapa hace referencia al direccionamiento estratégico, considerando a este como la etapa fundamental de esta generación de Balanced scorecard. (Massón, Guerra y Truno , 2006)

Según Alvarez et al. (como citó Fernández (2001), “el proceso de diseño del BSC inicia con la definición de la visión, misión y valores de la organización y a partir de ello se desarrolla la estrategia, que se representa a través del mapa estratégico. Un mapa estratégico es el conjunto de objetivos estratégicos que se relacionan a través de relaciones causa-efecto, ayudando a entender la coherencia entre los objetivos estratégicos y la estrategia de la organización".

Según los estudios de los expertos el futuro a corto plazo del BSC es el transformarse en una herramienta fundamental para la gestión del logro estratégico en las organizaciones, mediante este nuevo instrumento de gestión empresarial que permita adaptarse rápidamente a los frecuentes cambios de dirección estratégica causados por un entorno competitivo cada vez más demandante. (Álvarez, Chávez, y Moreno, 2012)

\section{Componentes del Balanced Scorecard}

- Análisis Causa Efecto: determinación de estrategias con medición de indicadores de desempeño. (Ayala, 2011) 
- Resultados Financieros: Aplicación de las perspectivas del BSC: Financiera, Clientes, Procesos Internos, y aprendizaje. (Ayala, 2011)

- Indicadores: La medición es el camino al cumplimiento de los objetivos, adaptados a cada entorno empresarial.

- Incentivos que impulsen el Cambio: Logrando la adaptabilidad a las estrategias y la mejora continua.

\section{Perspectivas del Balanced scorecard.}

Un método estratégico de Balanced scorecard de la empresa, se muestra más detallada mediante la definición de los objetivos e indicadores de las perspectivas, descritas a continuación:

Perspectiva Financiera. - dentro de la organización los indicadores financieros son fundamentales ya que muestran la realidad tal cual está sucediendo con la situación económica de la empresa.

Por ende, la organización debe evaluar constantemente en donde se debe realizar inversión que sirva de apoyo para el mejoramiento de los procesos y el crecimiento económica de la misma, mediante inversiones en nuevos proyectos que garanticen los resultados esperados. (Peralta, Erazo y Narvaez Zurita, 2019)

Perspectiva Clientes. - El cliente se transforma en un cliente estratégico de la empresa, donde aparte de generar ingresos monetarios también aporta para el crecimiento en el mercado con servicio y productos perfeccionados por sus exigencias. "La perspectiva del cliente permite a los directivos de unidades de negocio articular la estrategia de cliente basada en el mercado, que proporcionará unos rendimientos financieros futuros de categoría superior." (Kaplan y Norton).

Indicadores: Nivel de satisfacción de los clientes, participación del mercado, pedidos devueltos y cantidad de quejas. 
El cliente debe crear valor y estabilidad a la empresa, por lo tanto, la evaluación debe ser contante y siempre con miras a lograr la fidelización fortaleciendo su visión y misión.

Perspectiva procesos internos. - Para alcanzar los objetivos estratégicos de la empresa se debe partir de tener excelencia en ciertos procesos que dan vida a la empresa. Indicadores: Porcentaje de nuevos productos, devoluciones por productos defectuosos, tipos de fabricación y tiempo de respuesta al cliente.

Analizar los procesos internos de la empresa se vuelve primordial ya que se pueden mantener los que generan resultados positivos, mejorar los que requieren y descartar los que no reflejan los resultados estipulados. Con una buena gestión de los procesos se garantiza generar la ventaja competitiva para el mayor éxito de la empresa.

Perspectiva Formación y Crecimiento. Esta perspectiva es fundamental para la ejecución de las estrategias para conseguir los objetivos planteados direccionando a resultados a largo plazo, siendo constantes en el cambio y mejora continua logrando establecer una estructura organizacional idónea involucrando a las personas, sistemas y demás recursos.

Indicadores: Capital humano, sistema de infraestructura, clima organizacional.

El Balanced Scorecard sugiere que estas perspectivas abarcan todos los procesos necesarios para el correcto funcionamiento de una empresa y deben ser considerados en la definición de los indicadores. 


\section{Perpectivas del Balanced scorecard}

Figura 1

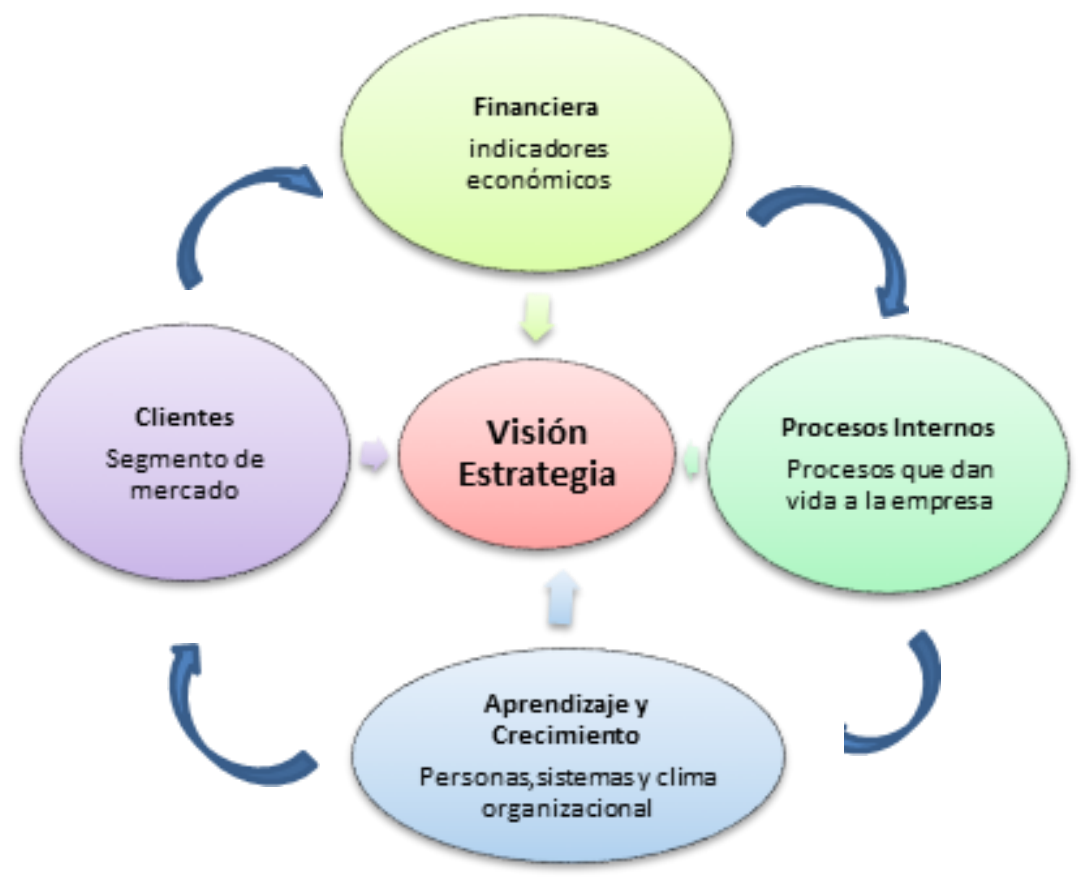

Fuente: Álvarez, Chavez y Moreno (2012).

El cuadro de mando integral permitió a las primeras organizaciones alinear sus recursos: tanto humanos, materiales y tecnológicos conjuntamente con los directivos para garantizar la eficiencia organizacional. Es importante evaluar los indicadores planteados en el estudio para controlar sus avances, partiendo de las mediciones realizadas en las fechas establecidas mediante valor de alerta, precaución y meta deseada (Sanclemente, 2015). "El cuadro de mando integral proporciona un marco que permite describir y comunicar una estrategia de forma coherente y clara, ya que no se puede aplicar una estrategia que no se pueda describir;

a diferencia de lo que pasa en las finanzas, hay un marco establecido como libros de contabilidad, las cuentas de resultados y los balances para documentar un plan financiero, no existen marcos generalmente aceptados para describir la estrategia organizacional; hay tantas maneras de describir una estrategia como teóricos y metodológicas que se relacionan con ella. Estudios hechos a organizaciones que aplican exitosamente 
el Cuadro de Mando Integral han descubierto un modelo repetitivo en el que siempre se da ese enfoque y hay alineación de la estrategia." (Sanchéz, 2010, p. 131)

\section{Beneficios del Balanced Scorecard (BSC)}

EI BSC muestra una metodología que vincula a la estrategia de la empresa con la acción, y resultados a través de la alineación de los objetivos de las perspectivas: financiera, clientes, procesos internos y aprendizaje y desarrollo, obteniendo beneficios como alineación de la visión de la empresa, una adecuada comunicación del personal, fijación de estrategias, automatización de la información para la mejora de la capacidad de análisis para la toma de decisiones.

El enfoque sistémico y la gestión de procesos, junto a sus implicaciones, constituyen un denominador común del concepto del Control Interno y del modelo Balanced Scorecard que, además comparten una visión global de la entidad, para lograr resultados óptimos para la organización. (Ramón, Erazo, Narvaez y Ormaza, 2019).

\section{Control Interno}

La gerencia moderna con el afán de conseguir controles óptimos a los procesos, denomina que el control interno tiene una vinculación directa con el recorrido del día a día de la empresa hacia el logro de sus objetivos y metas; el control interno no puede existir si previamente no existen objetivos, metas e indicadores de rendimiento. (Meléndez, 2016, p. 20)

El informe SAC (Sistema de Auditoría y Control) define a un sistema de control interno como: un conjunto de procesos, funciones, actividades, subsistemas, y gente que son agrupados o conscientemente segregados para asegurar el logro efectivo de los objetivos y metas. Especificando que, "El control interno es un medio para alcanzar un fin y no un fin en sí mismo, lo llevan a cabo las personas que conducen en todos los niveles, no se trata solamente de manuales de organización y procedimientos, sólo puede aportar un grado de seguridad razonable y no la seguridad total para la conducción o consecución de los objetivos". (Salas, 2011, p. 14) 
Holmes (1994) define, "Al hablarse del control interno como un proceso, se hace referencia a una cadena de acciones extendida a todas las actividades, inherentes a la gestión e integrados a los demás procesos básicos de la misma: planificación, ejecución y supervisión. Estas acciones se hallan incorporadas a la infraestructura de la entidad, para influir en el cumplimiento de sus objetivos y apoyar sus iniciativas de calidad". en (Salas, 2011, p. 15). Como objetivos generales del control interno se mencionan los siguientes:

1. Suscitar la eficiencia en las operaciones y la calidad de los servicios que debe brindar la empresa. Este objetivo hace referencia a seguir todos los pasos para lograr el control de las operaciones programadas, a través de la medición y seguimiento a cada una de las actividades realizadas.

2. Proteger y conservar los recursos contra cualquier riesgo. Este objetivo esta direccionado netamente a las medidas adoptadas por la administración de la empresa para prevenir o detectar operaciones incorrectas que darían como resultado pérdidas a la empresa.

3. Cumplimientos de las normas. Este objetivo enmarca a que mediante la disposición de normas, reglamentos y leyes se deben cumplir con el uso correcto de los recursos de la empresa.

4. Elaboración de informes financieros válidos, confiables y presentados oportunamente. Es de suma importancia ya que mediante la información confiable y oportuna la administración toma las decisiones acertadas.

5. Promover el cumplimiento de los funcionarios por la misión y objetivos encargados.

6. Es importante mencionar los principios del control interno:

7. Establecer responsabilidades.

8. Operaciones y registros contables deben estar separados.

9. Mediar el cumplimiento de las operaciones realizadas.

10. Las transacciones comerciales deben ser responsabilidad de todos los involucrados en el proceso.

11. Las tareas deben ser respaldas todas por escrito 


\section{Una correcta selección del personal}

13. El periodo de vacaciones debe ser gozado por todo el personal.

14. Reconcomiendo al personal no solo en dinero.

15. Acceso a rotación y asensos al personal según su mérito propio.

16. Aplicar los principios de contabilidad generalmente aceptados.

17. Generar un excelente sistema de archivo.

Resaltando como componentes del sistema de control interno:

1. El ambiente de control.-entendiendose las buenas practicas principios, valores y buena conducta de todo el personal involucrado.

2. La evaluación de riesgos.- Identificar, analizar, evaluar y mitigar los elementos que pueden no permitir conseguir los objetivos de la empresa.

3. Actividades de control gerencial.- Con la finalidad de asegurar el cumplimiento de los objetivos la adminsitración debe implementar, manual de procedicimiento, reglamento interno, politicas y normas garantizando su aplicación.

4. Las actividades de prevención y monitoreo.- haciendo referencia a las acciones adoptadas para el buen desempeño de las funciones asignadas con su respectivo sistema de seguimiento.

5. Los sistemas de información y comunicación.- Resaltando que es de vital importancia la aplicación de un sistema automatizado para obtener información confiable, transparente y acertada de cada uno de los procesos de gestión.

6. El seguimiento de resultados.- Consiste en la revisión medición y evaluacion de los logros obtenidos mediante las medidas de control interno.

7. Los compromisos de mejoramiento.- realizar una autoevalucion de la gestión de cada persona, informar cualquier anomalia en caso de existir, demostrando el compromiso y responsabilidad para apoyar a la mejora y optimizacion de los recursos. (Alvarez, Chavez y Moreno, 2004) (Aquipucho, 2015, ps. 24, 25) 


\section{METODOLOGÍA}

La presente investigación se desarrolló bajo un diseño no experimental, sin que las variables sean manipuladas y se observó el proceso del control interno en un cotexto natural, la misma que según el enfoque se aplicó una investigación mixta, considerándose cuantitativa por haber utilizado la recolección de datos con base en la medición numérica y el análisis estadístico y mientras que la cualitativa se utilizó con el objetivo de afinar las preguntas de investigación o revelar nuevas interrogantes en el proceso de interpretación. Según el alcance: La Investigación fue descriptiva ya que se especificaron las propiedades y las características de las variables de estudio, transformándose en un diseño explicativo secuencial (Dexplis) con el propósito de expandir los resultados, y según la finalidad se aplicó el diseño transversal para mediante la utilización de recolección de datos en un tiempo único describir las variables y su incidencia.

Los métodos aplicados fueron inductivo-deductivo partiendo de casos particulares a conocimientos generales permitiendo así la exploración y descripción de todos los procesos de la empresa, vinculando también a lo histórico-lógico para conocer la causa-efectos de las pérdidas económicas, a través de métodos estadísticos que permitió la separación de las variables para ser analizadas de forma minuciosa y analítica cada elemento para luego ser integradas mediante la síntesis a través de las técnicas aplicadas como la encuesta con un cuestionario dirigido a los empleados involucrados en los procesos, y además con la revisión documental que permitió realizar búsquedas retrospectivas y recuperar el documento necesario para conocer su contenido y análisis la aplicación, se recabó información de fuentes secundarias como es Reglamento Interno, Manual de procedimientos, código de ética, perfil de cargos.

El universo de estudio fue conformado por: 74 empleados y 60 clientes más frecuentes y de altos montos de venta. 


\section{Cálculo de la muestra}

Tabla 1

\begin{tabular}{lccccccc}
\hline Estratos & $\mathbf{N}$ & $\mathbf{P}$ & $\mathbf{Q}$ & $\mathbf{P Q}$ & $\mathbf{N P Q}$ & $\mathbf{W}$ & $\mathbf{n i}$ \\
\hline Empleados & 74 & 0.05 & 0.95 & 0.05 & 3.52 & 0.55 & 26 \\
Clientes & 60 & 0.05 & 0.95 & 0.05 & 2.38 & 0.45 & 21 \\
Total & $\mathbf{1 3 4}$ & & & & 6.37 & & \\
\hline
\end{tabular}

Aplicación de la Fórmula:

$$
\begin{array}{ll}
\mathrm{n}=\frac{\sum_{\mathrm{P} Q} \mathrm{~N}}{\mathrm{ND+1/N} \Sigma} & \\
\mathrm{NPQ} & \\
\mathrm{n}=\frac{6.37}{0.0872+0.0475} & \mathbf{n i =} \begin{array}{r}
26 \\
21
\end{array}
\end{array}
$$

Dando como resultado una muestra de 26 empleados distribuidos así:

a) Encuestas dirigidas a: Gerentes de Ventas, Gerente de Producción, Gerente administrativo financiero, Contador general, Supervisor de la planta, Coordinador de bodega de producto terminado, Coordinador de las bodegas de materias primas e insumos.

b) Encuestas dirigidas a: 5 operarios de la planta sección revisado de productos, 5 operarios de las bodegas de producto terminado, 2 asistentes contables, 1 asistente de compras, 1 asistente de ventas, 5 agentes vendedores.

c) Encuestas dirigidas a: 21 clientes importantes.

Ficha de contenido: Se analizó el manual de procedimientos, y reglamento interno.

\section{RESULTADOS}

Para un mejor análisis e interpretación de los resultados se procedió a describir según las Dimensiones con sus indicadores más relevantes: 
El Ambiente de control considerándose el indicador de Valores éticos, el 14\% de los empleados no conocen que la empresa dispone de un código de ética aprobado, y el 32\% no están al tanto del contenido. Sin embargo, el 100\% de los jefes departamentales han receptado eficazmente el compromiso de la Dirección hacia la integridad y comportamiento ético y desarrollan una administración estratégica para el cumplimiento de su misión. El $43 \%$ de los empleados no están de acuerdo que la estructura organizacional es adecuada para el tamaño y las actividades de sus operaciones y con respecto a la existencia de una adecuada supervisión de las operaciones de los procesos de la empresa el $14 \%$ de los encuestados afirman no estar de acuerdo. Analizando el indicador-Auditoria, preocupa saber que el $29 \%$ de los empleados considera que la unidad no cuenta con el personal adecuado para la cobertura de revisión, y tanto solo el $57 \%$ de los empleados receptan oportunamente la información requeridas a las áreas funcionales.

La dimensión de Evaluación de riesgos, mediante el indicador Riesgos, el 100\% de los empleados confirman que la dirección promueve la cultura de riesgos, estableciendo claramente los participantes en la identificación de los mismos tanto externos como internos y fue también relevante que según los tipos de riesgos el $71 \%$ afirmó haber sufrido un fraude interno seguido de un $43 \%$ de fallas técnicas sobre ejecución y gestión de procesos, para lo cual la administración tomando en cuenta el indicador-operaciones han desarrollado sistemas de información para identificar los riesgos críticos propiciando la participación de los "dueños del proceso" y se concluye el análisis que, en la empresa a pesar de existir procedimientos y planes claramente emitidos y difundidos hay deficiencia en la delegación de funciones y análisis de perfiles de cargos para la ejecución de las actividades generando inconformidad del personal y falta de comunicación continua entre las áreas.

Los resultados basados en el análisis de la dimensión de evaluación de información revelan que el $57 \%$ de los encuestados no conocen que la empresa cuenta con reglamento interno y manual de procedimientos, y el 53\% tampoco conoce su contenido. El $29 \%$ de los encuestados afirman que no existe suficiente información confiable para el seguimiento de las estrategias y cuyos resultados son comparados con los objetivos y 
metas programadas; sin embargo, aseguraron que los archivos físicos (documentación) disponen de protección adecuada. Referente a la comunicación entre las áreas dentro de la empresa el 53\% mantiene comunicación directa con el Gerente General, el 100\% a través del jefe inmediato y el $74 \%$ de los empleados considera que hay buena comunicación entre todas las áreas de la empresa.

En cuanto a la dimensión Financiera, analizando a los Estados Financieros, el 100\% de los encuestados confirman que el sistema de contabilidad utilizado en la empresa integra información financiera, patrimonial y presupuestaria en un sistema único, común, oportuno y confiable, sin embargo, resultó que el 32\% de los empleados expresan que el área contable no realiza confirmación de saldos en las cuentas por cobrar, cuentas por pagar y saldos de inventarios.

En el caso de la dimensión Clientes bajo el indicador- la satisfacción del cliente, los encuestados expresaron en un $74 \%$ que la empresa no realiza ninguna actividad que le permite determinar la percepción de los clientes respecto a los productos y servicios que ofrece, Sin embargo, el $42 \%$ asegura que la respuesta a los reclamos de los clientes es oportuna, demostrando claramente que existe una deficiencia debido a que los empleados tienen falta de conocimiento de los procedimientos de medición. Desde el punto de vista del Cliente considera importante el tiempo de entrega, el empaque del producto, calidad del producto y el servicio de transporte.

La dimensión Procesos internos a través de su indicador evaluaciones, indica que el 29\% de los empleados manifiestan que no existe de procedimientos para evaluar específicamente la competencia profesional al momento de la incorporación del empleado a la empresa.

Innovación y formación. - en cuanto a la capacitación del personal el 71\% de los empleados indican que la empresa cuenta con un plan de capacitación en donde todos participan y que el mismo corresponde a los objetivos y necesidades de cada empleado. 
Revista Arbitrada Interdisciplinaria KOINONIA

Año IV. Vol IV. Nº. Julio - Diciembre 2019

Hecho el depósito de Ley: FA2016000010

ISSN: 2542-3088

FUNDACIÓN KOINONIA (F.K). Santa Ana de Coro. Venezuela.

Ana Cristina Chirinos Martínez; Rubén Ulises Perozo Martín; Freddy José Rodríguez Acasio

\section{Desarrollo del modelo Balanced scorecard para el mejoramiento del control interno} Considerando las etapas para la implementación de un Balanced scorecard se parte de un análisis del FODA, estableciendo cual es la situación del ambiente interno y externo de la empresa, obteniendo las fortalezas, debilidades, oportunidades y amenas que garantizan que los objetivos y estrategias futuras estén acorde al entorno.

\section{Matriz de factores internos}

\section{Tabla 2}

\begin{tabular}{|l|c|c|c|}
\hline \multicolumn{1}{|c|}{ Factores } & Peso & Calificación & $\begin{array}{c}\text { Calificación } \\
\text { Ponderada }\end{array}$ \\
\hline \multicolumn{1}{|c|}{ Debilidades } & $50 \%$ & & 0.10 \\
\hline $\begin{array}{l}\text { 1. Falta de comunicación a todo el personal de la empresa sobre la } \\
\text { existencia de un código de ética aprobado. }\end{array}$ & 0.05 & 2 & 0.12 \\
\hline 2. Falta de seguimiento a recomendaciones de auditorias internas & 0.12 & 1 & 0.18 \\
\hline $\begin{array}{l}\text { 3. Falta de personal en la unidad de auditoria interna para cubrir todas } \\
\text { las áreas de control. }\end{array}$ & 0.09 & 2 & 0.18 \\
\hline $\begin{array}{l}\text { 4. Falta de conocimiento del personal sobre las Políticas y } \\
\text { procedimientos establecidos en la empresa. }\end{array}$ & 0.09 & 2 & 0.20 \\
\hline $\begin{array}{l}\text { 5. Falta de conocimiento del personal de los indicadores y medidores } \\
\text { para el cumplimiento del objetivo de la empresa. }\end{array}$ & 0.10 & 2 & 0.05 \\
\hline $\begin{array}{l}\text { 6. La estructura organizacional no es adecuada para el tamaño y las } \\
\text { actividades de operación. }\end{array}$ & 0.05 & 1 & 0.48 \\
\hline Fortalezas & $50 \%$ & & 0.15 \\
\hline $\begin{array}{l}\text { 1. Desarrollo de una administración estratégica para el cumplimiento de } \\
\text { la misión y visión de la empresa }\end{array}$ & 0.12 & 4 & 2.62 \\
\hline $\begin{array}{l}\text { 2. Adecuada supervisión y monitoreo de las operaciones de la empresa } \\
\text { Totales }\end{array}$ & 0.09 & 3 & 0.32 \\
\hline $\begin{array}{l}\text { 3. Existe la unidad de auditoria interna } \\
\text { las operaciones de la empresa y disponible para cada usuario según } \\
\text { sus necesidades. }\end{array}$ & 0.09 & 4 & 0.08 \\
\hline $\begin{array}{l}\text { 5. Elaboración del Plan de trabajo } \\
\text { 6. Demostración de trabajo en equipo }\end{array}$ & 0.07 & 3 & 0.05 \\
\hline
\end{tabular}


Una vez identificado los factores de debilidades y fortalezas se colocó un peso por importancia y posteriormente se calificó cada factor siendo 4 de mayor fortaleza, 3 de menor Fortaleza, 2 mayor debilidad y 1 de debilidad menor. En el análisis factores internos el factor de fortaleza más relevante es el Desarrollo de una administración estratégica para el cumplimiento de la misión y visión de la empresa, y como debilidad relevante es la falta de conocimiento del personal sobre los indicadores y medidores para el cumplimiento del objetivo de la empresa.

\section{Matriz de factores externos}

\section{Tabla 3}

\begin{tabular}{|l|c|c|c|}
\hline \multicolumn{1}{|c|}{ Factores } & Peso & Calificación & $\begin{array}{c}\text { Calificación } \\
\text { Ponderada }\end{array}$ \\
\hline \multicolumn{1}{|c|}{ Amenazas } & $50 \%$ & & \\
\hline $\begin{array}{l}\text { 1. La respuesta a los reclamos de los clientes no es } \\
\text { oportuna. }\end{array}$ & 0.15 & 3 & 0.45 \\
\hline $\begin{array}{l}\text { 2. Existe retrasos en el despacho de los pedidos a los } \\
\text { clientes. }\end{array}$ & 0.15 & 4 & 0.6 \\
\hline $\begin{array}{l}\text { 3.- Existe inconsistencias en los productos entregados al } \\
\text { cliente. }\end{array}$ & 0.2 & 4 & 0.8 \\
\hline $\begin{array}{l}\text { 1. La empresa realizar actividades que le permite } \\
\text { determinar la percepción de los clientes con respecto a } \\
\text { los productos y servicios que ofrece. }\end{array}$ & 0.2 & 4 & 0.8 \\
\hline $\begin{array}{l}\text { 2. El cliente califica a los productos que ofrece la empresa } \\
\text { como productos de muy buena calidad. }\end{array}$ & 0.2 & 3 & 0.6 \\
\hline $\begin{array}{l}\text { 3.- El cliente percibe que la empresa cuenta con una } \\
\text { estructura organizacional adecuada. }\end{array}$ & 0.1 & 2 & 0.2 \\
\hline Totales & $100 \%$ & & 3.45 \\
\hline
\end{tabular}

Con las mismas condiciones del análisis anterior se estudió la matriz de factores externos obteniendo como resultado que del factor de amenaza de mayor impacto fue las inconsistencias en los productos entregados a los clientes y del factor de oportunidad hizo 
Revista Arbitrada Interdisciplinaria KOINONIA

Año IV. Vol IV. Nº. Julio - Diciembre 2019

Hecho el depósito de Ley: FA2016000010

ISSN: 2542-3088

FUNDACIÓN KOINONIA (F.K). Santa Ana de Coro. Venezuela.

Ana Cristina Chirinos Martínez; Rubén Ulises Perozo Martín; Freddy José Rodríguez Acasio

énfasis en que la empresa realiza actividades que le permiten determinar la percepción de los clientes con respecto a los productos y servicios que ofrece la empresa.

En base a estos resultados se procedió a elaborar el mapa estratégico aplicando cada perspectiva del Balance scorecard.

\section{Mapa estratégico}

Figura 2

Perspectivas

Mapa Estratégico

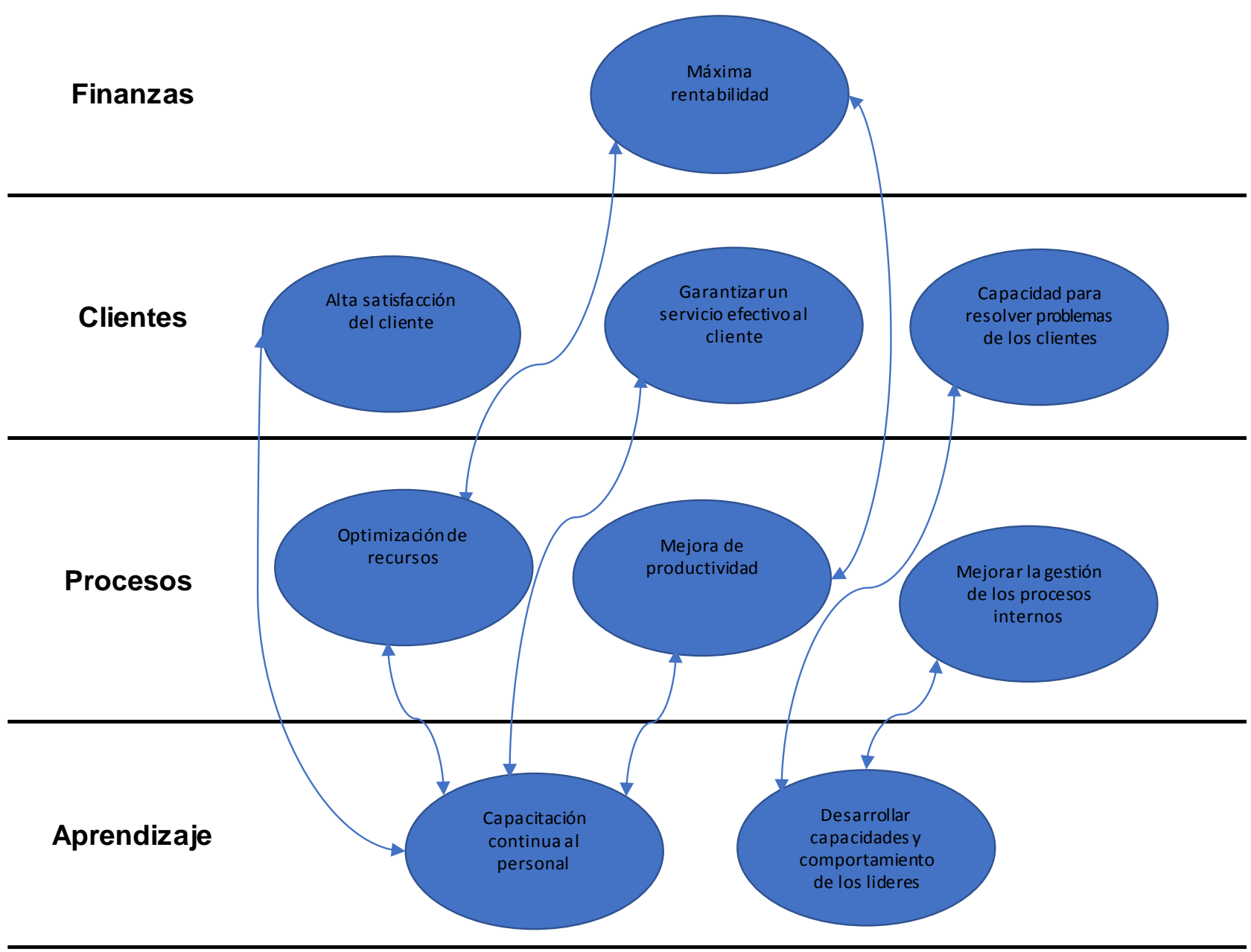


Revista Arbitrada Interdisciplinaria KOINONIA

Año IV. Vol IV. Nº 8 . Julio - Diciembre 2019

Hecho el depósito de Ley: FA2016000010

ISSN: 2542-3088

FUNDACIÓN KOINONIA (F.K). Santa Ana de Coro. Venezuela.

Ana Cristina Chirinos Martínez; Rubén Ulises Perozo Martín; Freddy José Rodríguez Acasio

Una vez determinado el mapa estratégico se estableció los objetivos estratégicos con sus

actividades y el indicador adecuado para la medición del cumplimiento de dichos objetivos con sus respectivos responsables. 
Revista Arbitrada Interdisciplinaria KOINONIA

Año IV. Vol IV. Nº ${ }^{\circ}$ Julio - Diciembre 2019

Hecho el depósito de Ley: FA2016000010

ISSN: 2542-3088

FUNDACIÓN KOINONIA (F.K). Santa Ana de Coro. Venezuela.

Ana Cristina Chirinos Martínez; Rubén Ulises Perozo Martín; Freddy José Rodríguez Acasio

\section{Estrategias del Balanced scorecard \\ Tabla 4}

Para una mejor interpretación de la aplicación del modelo de Balanced Scorecard se analiza de cada uno de los indicadores de las estrategias planteadas según como muestra en los cuadros siguientes:

\begin{tabular}{|c|c|c|c|c|c|c|}
\hline \multicolumn{7}{|c|}{ Balanced Scorecard } \\
\hline $\begin{array}{l}\text { PER } \\
\text { SP }\end{array}$ & Código & Objetivo estratégico & Actividad Estratégica & KPI & Meta & Responsable \\
\hline \multirow{2}{*}{ 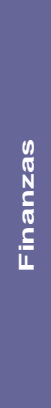 } & 11.01 & $\begin{array}{l}\text { Crear programas de incentivos y cambios } \\
\text { en la cultura organizacional desde los altos } \\
\text { mandos hasta los subalternos, es decir } \\
\text { involucrar a todo el personal en este } \\
\text { proceso. }\end{array}$ & $\begin{array}{l}\text { Crear parámetros de cumplimiento de las } \\
\text { actividades que sean claves para el logro de } \\
\text { los objetivos, ofreciendo un bono al } \\
\text { personal a cargo de dichas funciones, y } \\
\text { serán acreditados mensualmente dentro del } \\
\text { rol de pagos. }\end{array}$ & $\begin{array}{l}\% \text { De cumplimiento } \\
\text { mensual de las variables } \\
\text { consideradas para la } \\
\text { bonificación del personal }\end{array}$ & cumplimiento $>95 \%$ & Jefes de cada área \\
\hline & 2.01 & $\begin{array}{l}\text { Realizar de auditorias a las áreas críticas } \\
\text { enfocados a los principales riesgos } \\
\text { identificados. }\end{array}$ & $\begin{array}{l}\text { Elaboración de un plan de trabajo de cada } \\
\text { área a ser auditada en un periodo mensual. }\end{array}$ & $\begin{array}{l}\% \text { De cumplimiento del } \\
\text { plande trabajo }\end{array}$ & $K P I>95 \%$ & Auditor Interno \\
\hline \multirow{2}{*}{$\frac{0}{0}$} & 13.01 & $\begin{array}{l}\text { Determinar la percepción de los clientes } \\
\text { con respecto a los productos y servicios } \\
\text { que ofrece. }\end{array}$ & $\begin{array}{l}\text { Elaboración de un formulario para realizar } \\
\text { evaluaciones trimestrales a los clientes. }\end{array}$ & $\%$ De clientes satisfechos & $K P I>95 \%$ & $\begin{array}{c}\text { Gerente } \\
\text { comercial/asistente de } \\
\text { ventas }\end{array}$ \\
\hline & 14.01 & $\begin{array}{l}\text { Preparar al personal competente para } \\
\text { mantener informado al cliente y resolver } \\
\text { sus problemas. }\end{array}$ & $\begin{array}{l}\text { Elaboración de un plan de capacitación } \\
\text { mensual al personal sobre los productos y } \\
\text { servicios que ofrece la empresa }\end{array}$ & $\mid \begin{array}{l}\text { KPI } \quad \text { Cantidad de } \\
\text { problemas sin resolver }\end{array}$ & $\mathrm{KPI}<5$ trimestral & $\begin{array}{c}\text { Gerente } \\
\text { comercial/coordinador } \\
\text { de despachos. }\end{array}$ \\
\hline \multirow{2}{*}{$\begin{array}{l}n \\
0 \\
0 \\
0 \\
0 \\
0 \\
0 \\
0\end{array}$} & 15.01 & $\begin{array}{l}\text { Mantener informado a todo el personal } \\
\text { sobre las actualizaciones de los } \\
\text { procedimientos y políticas autorizadas por } \\
\text { el líder de área y Dirección General }\end{array}$ & $\begin{array}{l}\text { Elaboración de un cronograma de difusión } \\
\text { mensual, mediante charlas, y/o entregas de } \\
\text { documentos impresos a cada responsable } \\
\text { del proceso que esta involucrado. }\end{array}$ & $\begin{array}{l}\text { \% del personal que afirma } \\
\text { tener conocimiento } \\
\text { dichos manuales } \\
\text { procedimientos. }\end{array}$ & personal >95\% & Gerente de RRHH \\
\hline & 16.01 & $\begin{array}{l}\text { Generar beneficios de los cambios } \\
\text { implementados }\end{array}$ & $\begin{array}{l}\text { Medir el beneficio obtenido de cada cambio } \\
\text { o mejora implementada. }\end{array}$ & $\begin{array}{l}\text { \% de incremento en la } \\
\text { rentabilidad }\end{array}$ & $\begin{array}{l}\text { incremento en la } \\
\text { rentabilidad > } 5 \%\end{array}$ & $\begin{array}{c}\text { Gerente } \\
\text { Administrativo/financie } \\
\text { ro }\end{array}$ \\
\hline \multirow{2}{*}{$\begin{array}{l}\frac{0}{\sqrt{N}} \\
\frac{N}{0} \\
\frac{2}{0} \\
\frac{0}{4}\end{array}$} & 17.01 & $\begin{array}{l}\text { En base al plan de trabajo realizado de } \\
\text { manera objetiva, dar a conocer los } \\
\text { beneficios del área dentro de la } \\
\text { organización. }\end{array}$ & $\begin{array}{l}\text { Mediante reuniones trimestrales con los } \\
\text { jefes de áreas dar a conocer los beneficios } \\
\text { alcanzados y estos participar a sus } \\
\text { subalternos. }\end{array}$ & $\begin{array}{l}\text { Número de actas de cada } \\
\text { reunión con firma de } \\
\text { asistencia }\end{array}$ & personal >95\% & $\begin{array}{c}\text { Gerente } \\
\text { Administrativo/financie } \\
\text { ro }\end{array}$ \\
\hline & 18.01 & $\begin{array}{l}\text { Elaborarán los KPI's junto con los líderes } \\
\text { de área, para posteriormente realizar la } \\
\text { evaluación de los mismos. }\end{array}$ & $\begin{array}{l}\text { Hacer participe de la elaboración del plan } \\
\text { de trabajo con sus indicadores y mediciones } \\
\text { a cada uno de los involucrados en el } \\
\text { proceso y periódicamente deben ser } \\
\text { evaluados para medir el cumplimiento. }\end{array}$ & $\begin{array}{l}\% \text { de cumplimiento de las } \\
\text { metas. }\end{array}$ & cumplimiento $>95 \%$ & $\begin{array}{c}\text { Gerente de } \\
\text { producción/Gerente } \\
\text { Administrativo }\end{array}$ \\
\hline
\end{tabular}


Revista Arbitrada Interdisciplinaria KOINONIA

Año IV. Vol IV. Nº ${ }^{\circ}$ Julio - Diciembre 2019

Hecho el depósito de Ley: FA2016000010

ISSN: 2542-3088

FUNDACIÓN KOINONIA (F.K). Santa Ana de Coro. Venezuela.

Ana Cristina Chirinos Martínez; Rubén Ulises Perozo Martín; Freddy José Rodríguez Acasio

Indicador I1.01 - I2.01

Tabla 5

\begin{tabular}{|c|c|}
\hline Nombre del indicador & $\begin{array}{l}\text { \% De cumplimiento mensual de las variables consideradas } \\
\text { para la bonificación del personal }\end{array}$ \\
\hline Código & 11.01 \\
\hline Objetivo & $\begin{array}{l}\text { Crear programas de incentivos y cambios en la cultura } \\
\text { organizacional desde los altos mandos hasta los } \\
\text { subalternos, es decir involucrar a todo el personal en este } \\
\text { proceso. }\end{array}$ \\
\hline Responsable del Indicador & Jefes de cada área \\
\hline Nivel de comparación & Indicador Meta \\
\hline Periodicidad de actualización & Mensual \\
\hline Definición Operacional & $\begin{array}{l}\text { Crear parámetros de cumplimiento de las actividades que } \\
\text { sean claves para el logro de los objetivos, ofreciendo un } \\
\text { bono al personal a cargo de dichas funciones, y serán } \\
\text { acreditados mensualmente dentro del rol de pagos. }\end{array}$ \\
\hline Unidad de medida & $\%$ \\
\hline Meta & cumplimiento $>95 \%$ \\
\hline \multirow{3}{*}{ Resultado } & $\mid 1.01 \geq 80 \%$ \\
\hline & $80 \%<11.01>60 \%$ \\
\hline & $11.01<60 \%$ \\
\hline Interpretación & $\begin{array}{l}\text { Permite evaluar el cumplimiento de las actividades } \\
\text { estratégicas a cargo de cada funcionario. }\end{array}$ \\
\hline
\end{tabular}

\begin{tabular}{|c|c|}
\hline Nombre del indicador & $\%$ De cumplimiento del plan de trabajo \\
\hline Código & 12.01 \\
\hline Objetivo & $\begin{array}{l}\text { Realizar de auditorias a las áreas criticas enfocados a } \\
\text { los principales riesgos identificados. }\end{array}$ \\
\hline Responsable del Indicador & Auditor Interno \\
\hline Nivel de comparación & Indicador Meta \\
\hline Periodicidad de actualización & Mensual \\
\hline Definición Operacional & $\begin{array}{l}\text { Eaboración de un plan de trabajo de cada área a ser } \\
\text { auditada en un periodo mensual. }\end{array}$ \\
\hline Unidad de medida & $\%$ \\
\hline Meta & $K P \mid>95 \%$ \\
\hline \multirow{3}{*}{ Resultado } & $12.01>95 \%$ \\
\hline & $95 \%<12.01>75 \%$ \\
\hline & $\mid 2.01<75 \%$ \\
\hline Interpretación & Identifica y controla los procesos críticos de cada área. \\
\hline
\end{tabular}


Revista Arbitrada Interdisciplinaria KOINONIA

Año IV. Vol IV. Nº ${ }^{\circ}$ Julio - Diciembre 2019

Hecho el depósito de Ley: FA2016000010

ISSN: 2542-3088

FUNDACIÓN KOINONIA (F.K). Santa Ana de Coro. Venezuela.

Ana Cristina Chirinos Martínez; Rubén Ulises Perozo Martín; Freddy José Rodríguez Acasio

\section{Indicador 13.01 - 14.01}

\section{Tabla 6}

\begin{tabular}{|c|c|}
\hline Nombre del indicador & $\%$ De clientes satisfechos \\
\hline Código & 13.01 \\
\hline Objetivo & $\begin{array}{l}\text { Determinar la percepción de los clientes con respecto a los } \\
\text { productos y servicios que ofrece. }\end{array}$ \\
\hline Responsable del Indicador & Gerente comercial/asistente de ventas \\
\hline Nivel de comparación & Indicador Meta \\
\hline Periodicidad de actualización & trimestral \\
\hline Definición Operacional & $\begin{array}{l}\text { Eaboración de un formulario para realizar evaluaciones } \\
\text { trimestrales a los clientes. }\end{array}$ \\
\hline Unidad de medida & $\%$ \\
\hline Meta & $K P \mid>95 \%$ \\
\hline \multirow{3}{*}{ Resultado } & $13.01>95 \%$ \\
\hline & $95 \%<13.01>75 \%$ \\
\hline & $13.01<75 \%$ \\
\hline Interpretación & $\begin{array}{l}\text { Permite medir el porcentaje de clientes satisfechos } \\
\text { asegurando la fidelización de los mismos. }\end{array}$ \\
\hline
\end{tabular}

\begin{tabular}{|c|c|}
\hline Nombre del indicador & KPl $=$ Cantidad de problemas sin resolver \\
\hline Código & 14.01 \\
\hline Objetivo & $\begin{array}{l}\text { Preparar al personal competente para mantener } \\
\text { informado al cliente y resolver sus problemas. }\end{array}$ \\
\hline Responsable del Indicador & Gerente comercial/coordinador de despachos. \\
\hline Nivel de comparación & Indicador Meta \\
\hline Periodicidad de actualización & Mensual \\
\hline Definición Operacional & $\begin{array}{l}\text { Elaboración de un plan de capacitación mensual a } \\
\text { personal sobre los productos y servicios que ofrece la } \\
\text { empresa }\end{array}$ \\
\hline Unidad de medida & Unidad \\
\hline Meta & 5 reclamos \\
\hline \multirow{3}{*}{ Resultado } & $4.01 \leq 4$ \\
\hline & $8>11.01>4$ \\
\hline & $4.01>8$ \\
\hline Interpretación & $\begin{array}{l}\text { Mide el número de reclamos de los clientes no atendidos } \\
\text { oportuna y correctamente. }\end{array}$ \\
\hline
\end{tabular}

\section{Indicador I5.01 - I6.01}

\section{Tabla 7}

\begin{tabular}{|c|c|}
\hline Nombre del indicador & $\begin{array}{l}\% \text { del personal que afirma tener conocimiento de dichos } \\
\text { manuales y procedimientos. }\end{array}$ \\
\hline Código & 15.01 \\
\hline Objetivo & $\begin{array}{l}\text { Mantener informado a todo el personal sobre las } \\
\text { actualizaciones de los procedimientos y políticas } \\
\text { autorizadas por el líder de área y Dirección General }\end{array}$ \\
\hline Responsable del Indicador & Gerente de RRHH \\
\hline Nivel de comparación & Indicador Meta \\
\hline Periodicidad de actualización & mensual \\
\hline Definición Operacional & $\begin{array}{l}\text { Elaboración de un cronograma de difusión mensual, mediante } \\
\text { charlas, y/o entregas de documentos impresos a cada } \\
\text { responsable del proceso que esta involucrado. }\end{array}$ \\
\hline Unidad de medida & $\%$ \\
\hline Meta & personal $>95 \%$ \\
\hline \multirow{3}{*}{ Resultado } & $15.01>95 \%$ \\
\hline & $95 \%<15.01>75 \%$ \\
\hline & $15.01<75 \%$ \\
\hline Interpretación & $\begin{array}{l}\text { Permite medir el \% del personal que tiene conocimiento sobre } \\
\text { las políticas y procedimientos de la empresa. }\end{array}$ \\
\hline
\end{tabular}

\begin{tabular}{|l|l|}
\hline Nombre del indicador & \% de incremento en la rentabilidad \\
\hline Código & 16.01 \\
\hline \multirow{2}{*}{ Objetivo } & Generar beneficios de los cambios implementados \\
\hline Responsable del Indicador & Gerente Administrativo/financiero \\
\hline Nivel de comparación & Indicador Meta \\
\hline Periodicidad de actualización & Mensual \\
\hline \multirow{2}{*}{ Definición Operacional } & $\begin{array}{l}\text { Medir el beneficio obtenido de cada cambio o mejora } \\
\text { implementada. }\end{array}$ \\
\hline Unidad de medida & \multicolumn{2}{|c|}{ incremento en la rentabilidad $>5 \%$} \\
\hline Meta & \multicolumn{2}{|c|}{$16.01>5 \%$} \\
\hline \multirow{2}{*}{ Resultado } & \multicolumn{2}{|c|}{$16.01<3 \%$} \\
\cline { 2 - 3 } & $\begin{array}{l}\text { Mide el incremento de la rentabilidad resultado de los } \\
\text { beneficios de realizar cambios o mejoras mediante el } \\
\text { cumplimiento del plan de trabajo. }\end{array}$ \\
\hline \multirow{2}{*}{ Interpretación } &
\end{tabular}


Revista Arbitrada Interdisciplinaria KOINONIA

Año IV. Vol IV. Nº. Julio - Diciembre 2019

Hecho el depósito de Ley: FA2016000010

ISSN: 2542-3088

FUNDACIÓN KOINONIA (F.K). Santa Ana de Coro. Venezuela.

Ana Cristina Chirinos Martínez; Rubén Ulises Perozo Martín; Freddy José Rodríguez Acasio

Indicador 16.01 - 18.01

Tabla 8

\begin{tabular}{|c|c|}
\hline Nombre del indicador & incremento en la rentabilidad > 5\% \\
\hline Código & 17.01 \\
\hline Objetivo & $\begin{array}{l}\text { En base al plan de trabajo realizado de manera objetiva, dar } \\
\text { a conocer los beneficios del área dentro de la organización. }\end{array}$ \\
\hline Responsable del Indicador & Gerente Administrativo/financiero \\
\hline Nivel de comparación & Indicador Meta \\
\hline Periodicidad de actualización & Trimestral \\
\hline Definición Operacional & $\begin{array}{l}\text { Mediante reuniones trimestrales con los jefes de áreas dar a } \\
\text { conocer los beneficios alcanzados y estos participar a sus } \\
\text { subalternos. }\end{array}$ \\
\hline Unidad de medida & $\%$ \\
\hline Meta & personal $>95 \%$ \\
\hline \multirow{3}{*}{ Resultado } & $17.01>95 \%$ \\
\hline & $95 \%<17.01>75 \%$ \\
\hline & $17.01<75 \%$ \\
\hline Interpretación & $\begin{array}{l}\text { Medir el \% del personal con respecto al total de empleados } \\
\text { que afirme tener conocimiento de los beneficios alcanzados } \\
\text { resultado del cumplimiento del plan de trabajo. }\end{array}$ \\
\hline
\end{tabular}

\begin{tabular}{|c|c|}
\hline Nombre del indicador & $\%$ de cumplimiento de las metas. \\
\hline Código & 18.01 \\
\hline Objetivo & $\begin{array}{l}\text { Eaborarán los KPI's junto con los líderes de área, para } \\
\text { posteriormente realizar la evaluación de los mismos. }\end{array}$ \\
\hline Responsable del Indicador & Gerente de producción/Gerente Administrativo \\
\hline Nivel de comparación & Indicador Meta \\
\hline Periodicidad de actualización & Mensual \\
\hline Definición Operacional & $\begin{array}{l}\text { Hacer participe de la elaboración del plan de trabajo con } \\
\text { sus indicadores y mediciones a cada uno de los } \\
\text { involucrados en el proceso y periódicamente deben ser } \\
\text { evaluados para medir el cumplimiento. }\end{array}$ \\
\hline Unidad de medida & $\%$ \\
\hline Meta & cumplimiento $>95 \%$ \\
\hline \multirow{3}{*}{ Resultado } & $18.01>95 \%$ \\
\hline & $95 \%<18.01>75 \%$ \\
\hline & $18.01<75 \%$ \\
\hline Interpretación & Medir el \% de cumplimiento de las metas planteadas. \\
\hline
\end{tabular}

\section{CONCLUSIONES}

- La aplicación del modelo Balanced scorecard implementado según la necesidad empresarial constituyen una herramienta administrativa imprescindible para lograr una administración eficiente que genere flujo de información y sobre todo beneficios monetarios.

- Incrementar la utilidad de la empresa mediante la implementación de bonificaciones adicionales basados en el desempeño del trabajador, genera excelentes resultados, siempre y cuando sean aplicados cumpliendo lo estipulado en el Art. 81 del código de trabajo, lo cual en Indalum se aplica correctamente.

- Basados en los principios de contabilidad generalmente aceptados resaltando la objetividad de la información, Indalum cuenta con un sistema contable automatizado donde se registra todas las operaciones cuando sucede y con la certeza de 
su valor y hecho subyacente logrando una presentación razonable de los estados financieros.

- Basados en la revisión de áreas seleccionadas, se ha detectado la comunicación deficiente en el control interno, en los procedimientos de contabilidad de acuerdo a lo establecido en la NIA No. 265.

- Los estados financieros están presentados razonablemente, en todos los aspectos materiales, la situación financiera de Indalum y los resultados de sus operaciones están de acuerdo a las Normas Internacionales de Información Financiera.

- Es de vital importancia que todo el personal desde los altos directivos hasta el personal operativo tenga conocimiento de los objetivos estratégicos de la empresa y trabajen en con un propósito común para lograr obtener las metas planteadas.

\section{REFERENCIAS CONSULTADAS}

1. Alvarez Medina, M., Chavez Rivera, M., y Moreno Velarde, S. (2004). El Balanced Scorecard, una herramienta para planeación estrategica. Sonora: Profesores investigadors del Departamento de Contaduría y Finanzas del Instituto Tecnológico de Sonora.

2. Álvarez, A. (2014). La medición de la eficiencia y la productividad. Madrid: Ediciones Pirámide.

3. Alvarez, M., Chavez, M., y Moreno, S. (2012). Planeación Estrategica Balanced Scoredcard. Sonora.

4. Andrade, P. (2014). Balanced Scorecard como modelo de gestión para la planeación, segumiento y evaluación de actividades que conlleven al desarrollo de la competencia cuidadana a atraves de los gestores de paz en el INEM Francisco de Paula Santander. Bogotá: Universidad Libre .

5. Aparicio, C., \& Moreno, H. (2011). Calidad de la cartera crediticia bancaria y el ciclo económico: una mirada. Lima: SUPERINTENDENCIA DE BANCA, SEGUROS Y ADMINISTRADORAS.

6. Aquipucho, L. (2015). Control interno y su influencia en Iso procesos de adquisiciones y contrataciones de la municipalidd districtal carmen de la lengua Reynoso . Lima - Perú: Universidad Nacional Mayor de San Marcos. 
7. Argueta, H., Flores, A., y Renderos, C. (2010). Diseño de herramientas de gestión administratia y financiera para Agencias de viajes en el Salvador. El Salvador.

8. Ayala, M. (2011). Balanced Scoredcard Importncia en los sistemas de Calidad. Bogotá.

9. Choez, W. (2017). El sistema de control interno como instrumemto de gestion para la adecuada toma de dicisiones en la empresa servicentro primavera SRL. Cajamarca - Peru: Universidad Nacional de Cajamarca .

10. Manzo, J. (2013). Balanced scorecard, herramienta de medición en una empresa PYME de la industria de la constricción. Tlaquepaque, Jalisto: Instituto Tecnologico y de estudios superiores de Occidente.

11. Martínez, L., \& El Kadi, O. (2019). Logística Integral y Calidad Total, Filosofía de Gestión Organizacional orientadas al cliente. Revista Arbitrada Interdisciplinaria Koinonía, 4(7), 202-232. doi:http://dx.doi.org/10.35381/r.k.v4i7.201

12. Masson, J., y Truñó, J. (2006). La Cuarta Generación Balanced Scoredcrd. Barcelona.

13. Meléndez, J. (2016). Control Interno. Ancash - Perú: Universidad Católica Los Angeles de Chimbote.

14. Montoya, C. (2011). El Balanced scorecard como herramienta de evaluación en la gestión administrativa. Medellín-Colombia: Visión de Futuro.

15. Ortega, A., Padilla, S., y Ruz, A. (2017). Nivel de importacia del control interno de los inventarios dentro del marco conceptual de una empresa. Comlombia: Lideraz Estratégico.

16. Peralta Llivipuma, M. R., Erazo Alvarez, J. C., \& Narvaez Zurita, C. I. (2019). Cuadro de mando integral, enfoque estratégico al proceso administrativo y educativo. Visionario Digital, 130.

17. Ramón Saquicela, J. S., Erazo Alvarez, J. C., Narvaez Zurita, C. I., \& Ormaza Andrade, J. E. (2019). Plan estratégico financiero para la óptima gestión de los ingresos en las cooperativas de ahorro y crédito del sector financiero popular y solidario. Visionario Digital, 225. 
18. Sanclemente, J. (2015). Balanced scorecard en el departamento administrativo financiero. Estudio caso: empresa de transportes del grupo internacional Mexicano. Cali-Colombia: Fundación Universitaria Católica Lumen Gentium.

\section{REFERENCES CONSULTED}

1. Alvarez Medina, M., Chavez Rivera, M., and Moreno Velarde, S. (2004). The Balanced Scorecard, a tool for strategic planning. Sonora: Research professors of the Accounting and Finance Department of the Sonora Institute of Technology.

2. Álvarez, A. (2014). The measurement of efficiency and productivity. Madrid: Pyramid Editions.

3. Alvarez, M., Chavez, M., and Moreno, S. (2012). Balanced Scoredcard Strategic Planning. Sonora

4. Andrade, P. (2014). Balanced Scorecard as a management model for planning, monitoring and evaluation of activities that lead to the development of urban competence through peace managers in the INEM Francisco de Paula Santander. Bogotá: Free University.

5. Aparicio, C., \& Moreno, H. (2011). Quality of the bank loan portfolio and the economic cycle: a look. Lima: SUPERINTENDENCE OF BANKING, INSURANCE AND ADMINISTRATORS.

6. Aquipucho, L. (2015). Internal control and its influence on the procurement and contracting processes of the Carmen district municipality of the Reynoso language. Lima - Peru: National University of San Marcos.

7. Argueta, H., Flores, A., and Renderos, C. (2010). Design of administrative and financial management tools for travel agencies in El Salvador. The Savior.

8. Ayala, M. (2011). Balanced Scoredcard Importance in Quality systems. Bogota

9. Choez, W. (2017). The internal control system as a management instrument for the proper decision making in the company servicentro primavera SRL. Cajamarca Peru: National University of Cajamarca.

10. Manzo, J. (2013). Balanced scorecard, measurement tool in a SME company in the constriction industry. Tlaquepaque, Jalisto: Technological and Higher Education Institute of the West. 
11. Martínez, L., \& El Kadi, O. (2019). Integral Logistics and Total Quality, Customer Management Organizational Management Philosophy. Interdisciplinary Arbitrated Review Koinonía, 4 (7), 202-232. doi: http: //dx.doi.org/10.35381/r.k.v4i7.201

12. Masson, J., and Truñó, J. (2006). The Fourth Generation Balanced Scoredcrd. Barcelona.

13. Meléndez, J. (2016). Internal control. Ancash - Peru: Los Angeles de Chimbote Catholic University.

14. Montoya, C. (2011). The Balanced scorecard as an evaluation tool in administrative management. Medellin-Colombia: Vision of the Future.

15. Ortega, A., Padilla, S., and Ruz, A. (2017). Level of importance of internal inventory control within the conceptual framework of a company. Comlombia: Strategic Leadership.

16. Peralta Llivipuma, M. R., Erazo Alvarez, J. C., \& Narvaez Zurita, C. I. (2019). Integral scorecard, strategic approach to the administrative and educational process. Digital Visionary, 130.

17. Ramón Saquicela, J. S., Erazo Alvarez, J. C., Narvaez Zurita, C. I., \& Ormaza Andrade, J. E. (2019). Strategic financial plan for the optimal management of income in savings and credit cooperatives of the popular and solidarity financial sector. Digital Visionary, 225.

18. Sanclemente, J. (2015). Balanced scorecard in the financial administrative department. Case study: transport company of the Mexican international group. Cali-Colombia: Lumen Gentium Catholic University Foundation.

(C2019 por los autores. Este artículo es de acceso abierto y distribuido según los términos y condiciones de la licencia Creative Commons Atribución-NoComercial-Compartirlgual 4.0 Internacional (CC

BY-NC-SA 4.0) (https://creativecommons.org/licenses/by-nc-sa/4.0/). 